\title{
Measurement of endothelial function and its clinical utility for cardiovascular risk
}

\author{
Mo Al-Qaisi' \\ Rajesh K Kharbanda' \\ Tarun K Mittal' \\ Ann E Donald ${ }^{2}$
}

'Royal Brompton and Harefield NHS Trust, London, UK; ${ }^{2}$ Institute of Child Health and Great Ormond Street Hospital, NHS Trust, London, UK
Correspondence: Mo Al-Qaisi Suite 280, 28 Old Brompton Road, London SW7 3SS, United Kingdom Tel +447968 016315

Fax +44 2077364573

Emailmrcgp74@yahoo.co.uk

\begin{abstract}
Over the past two decades, the central role of the endothelium in the initiation, progression, and clinical sequelae of atherosclerosis has been increasingly recognized. Assessment of the pathobiology of the endothelium and its ability to act as a potential therapeutic target remains an area of active research interest. Whilst endothelial function has been shown to be a marker for risk of cardiovascular events in high-risk groups, there remains considerable debate about the most appropriate way to assess this. We discuss the different clinical methods to assess endothelial function, focusing on flow-mediated dilatation (FMD) of the brachial artery, highlighting the importance of using a standardized methodology, as well as discussing the clinical limitations of using FMD in individuals.
\end{abstract}

Keywords: endothelial function, flow-mediated dilatation, clinical utility, cardiovascular risk

\section{Physiology of the endothelium}

The single layer of endothelial cells lining the blood vessels produces autoimmune, endocrine, and paracrine factors (Schechter and Gladwin 2003). Endothelial cells are involved in the modulation of platelet activation, leukocyte adhesion, thrombosis, and the regulation of vasomotor tone by production of vasoactive substances, particularly nitric oxide (NO), endothelin, prostacyclin, and angiotensinogen (Furchgott and Zawadzki 1980; Smiesko et al 1985; Pohl et al 1986). By producing nitric oxide, the endothelium has a central homeostatic role in the vascular system, allowing organ perfusion to be matched to the cardiac output (Rubanyi et al 1986; Corson et al 1996). The homeostatic, vasodilator function of the endothelium (mediated by NO) has become a useful target for the indirect assessment of NO bioavailability.

Nitric oxide is a key antiatherogenic molecule produced by the healthy endothelium (Cooke and Dzau 1997; Kharbanda and Deanfield 2001), however its protective effects are lost if the endothelium is damaged (Forstermann and Munzel 2006).

\section{Atherosclerosis}

Atherosclerosis is a systemic arterial disease whereby areas of damaged endothelium promote entry of circulating inflammatory cells and set up a process of lipid deposition and fibrosis. Over a number of years the lesions can progress to cause luminal narrowing, which can reduce blood flow, or can form a nidus for thrombus formation and manifest as acute cardiovascular syndromes such as myocardial infarction or stroke.

Conventional risk factors for atherosclerosis can be increasingly identified in the general population but targeting those likely to develop disease and maximizing the benefits of any intervention has not been realized. Risk factors associated with the development of atherosclerosis include age, smoking, hypertension, diabetes and family history, but despite large scale epidemiological studies, approximately half the burden 
of atherosclerotic cardiovascular disease is unexplained by conventional risk factors. Patients with manifest complications of atherosclerosis and those who are at high risk of future cardiovascular events require aggressive treatment of risk factors. Risk factor modification is associated with improved outcome (Ford et al 2007).

Disturbances in vascular biology provide a pathophysiological basis to identify individuals at future increased risk of acute cardiovascular events (Celermajer et al 1994; Halcox et al 2002; Shimbo et al 2007). Anatomical assessment of coronary atherosclerosis by intravascular ultrasound or assessment of carotid intima media thickness are currently accepted as suitable surrogate clinical trial endpoints by the Food and Drug Administration in the United States for cardiovascular intervention trials (Sankatsing et al 2005; Nicholls et al 2006). Some studies have suggested a link between improved vascular function and outcome (Suessenbacher et al 2006), however this remains to be confirmed in larger studies, and the question of whether targeted improvement of endothelial function translates into clinical benefit has not been answered.

Primary prevention is an altogether more controversial area. Atherosclerosis takes many decades to develop and can be considered a disease of childhood (Leeson et al 1997; Reilly et al 2005). Prevention of early atherosclerosis at its preclinical stage would have major global health impact. However work is still emerging on the approach to its identification and treatment in the young (Celermajer and Ayer 2006).

\section{Measures of endothelial function}

There are many techniques for assessing endothelial function. These techniques can be either invasive or noninvasive, and assess different aspects of pathobiology (1). For the assessment of pre-clinical disease, the ideal technique for measuring endothelial function must be noninvasive, reliable, reproducible, cheap, and easy to perform (Deanfield et al 2007).

\section{Invasive techniques}

Vasoactive agents are delivered via intra-arterial infusion, whilst the response is measured with high resolution ultrasound or strain gauge plethysmography. In addition, intravascular infusions of vasoactive stimulants can be combined with intravascular ultrasound. For example, intravascular coronary flow-mediated dilatation (FMD) studies (Halcox et al 2002) or studies of radial artery grafts used for coronary bypass surgery (Chong et al 2006).
Table I Methods for measuring endothelial function

\begin{tabular}{ll}
\hline Technique & Method \\
\hline Invasive & $\begin{array}{l}\text { Intra-arterial ACh or edothelin infusion and strain } \\
\text { gauge plethysmography (or high resolution ultrasound) } \\
\text { Intravascular US studies of epicardial coronary arteries }\end{array}$ \\
Noninvasive & US FMD of the brachial artery \\
& Flow-mediated MRI \\
& Pulse wave analysis (PWA) applanation tonometry \\
& with inhaled salbutamol (B $\mathrm{B}_{2}$ agonist for global \\
& endothelial NO release) \\
& $\begin{array}{l}\text { Pulse contour analysis (PCA) digital } \\
\text { photoplethysmography with inhaled salbutamol } \\
\text { (B. }\end{array}$ \\
Microvascular agonist for global endothelial NO release) & Laser Doppler skin flowmetry \\
& Digital pulse amplitude tonometry (PAT)
\end{tabular}

Abbreviations: FMD, flow-mediated dilation; MRI, magnetic resonance imaging; NO, nitric oxide; US, United States of America.

\section{Noninvasive techniques}

Noninvasive methods of measuring endothelial function include ultrasound FMD, salbutamol-mediated endothelial function measured by pulse wave analysis (PWA) or pulse contour analysis (PCA), flow-mediated magnetic resonance imaging (MRI), laser Doppler flowmetry, and flow-mediated pulse amplitude tonometry (PAT).

Noninvasive ultrasound FMD of the brachial artery is the most widely used method for both small and large population studies of adults and children. FMD measured by MRI has been validated against ultrasound for measuring endothelial function. Although it is less operator-dependent than ultrasound, and allows simultaneous assessment of peripheral and central endothelial function, it is more expensive, less widely available, and has the general disadvantages of MR-based techniques (Leeson et al 2006).

Pulse wave analysis and PCA with salbutamol are more recent methods which may be cheaper than ultrasound FMD but require larger sample sizes as there is more variability; in addition they have been shown to be less reliable in children (Donald et al 2006).

\section{Microvascular techniques}

Laser Doppler skin flowmetry has been correlated with endothelial function of the brachial artery (Hansell et al 2004). It has been used as a research tool (Strain et al 2005), however it remains uncertain if this technique reflects the same pathobiology as that of conduit vessel endothelial dysfunction.

Digital PAT is also a recent technology which has been shown to correlate with microvascular endothelial dysfunction of the coronary arteries (Bonetti et al 2004). More data is required on its reproducibility and its utility for assessment of endothelial function. 


\section{Flow-mediated dilatation of the brachial artery}

Noninvasive brachial artery assessment of FMD has emerged as an important research tool for assessment of endothelial function. Measuring endothelial function in the brachial artery may offer a noninvasive measure of preclinical cardiovascular risk in populations with and without obvious risk factors (Celermajer et al 1994; Anderson 2007), although the results of larger outcome studies are still emerging (Shimbo et al 2007; Yeboah et al 2007). Preclinical detection of endothelial dysfunction will enable interventions to be targeted and monitored at an early stage in the disease process.

Brachial artery FMD has been utilized for measurement of endothelial function for over 15 years (Anderson and Mark 1989; Celermajer et al 1992). FMD is the measurement of transient changes in brachial artery diameter in response to shear stress. An increase in flow in the brachial artery is achieved by inflation of a pneumatic cuff (placed on the forearm, distal to the ultrasound imaging site) to suprasystolic pressure for 5 minutes (see Figure 1). On deflation of the cuff, the increased flow results in shear stress which activates endothelial nitric oxide synthase to release NO via the L-arginine pathway. The NO diffuses to the smooth muscle cells causing them to relax resulting in vasodilatation. FMD is measured as the percentage change in brachial artery diameter from baseline in response to the increased flow. Noninvasive ultrasound FMD of the brachial artery is now widely quoted in the literature, however, standardization of its methodology must precede its clinical application.

\section{Towards a standard methodology for ultrasound FMD of the brachial artery}

Despite being the most frequently used technique, a closer look at the literature reveals that there are wide variations in mean FMD between studies of the same populations
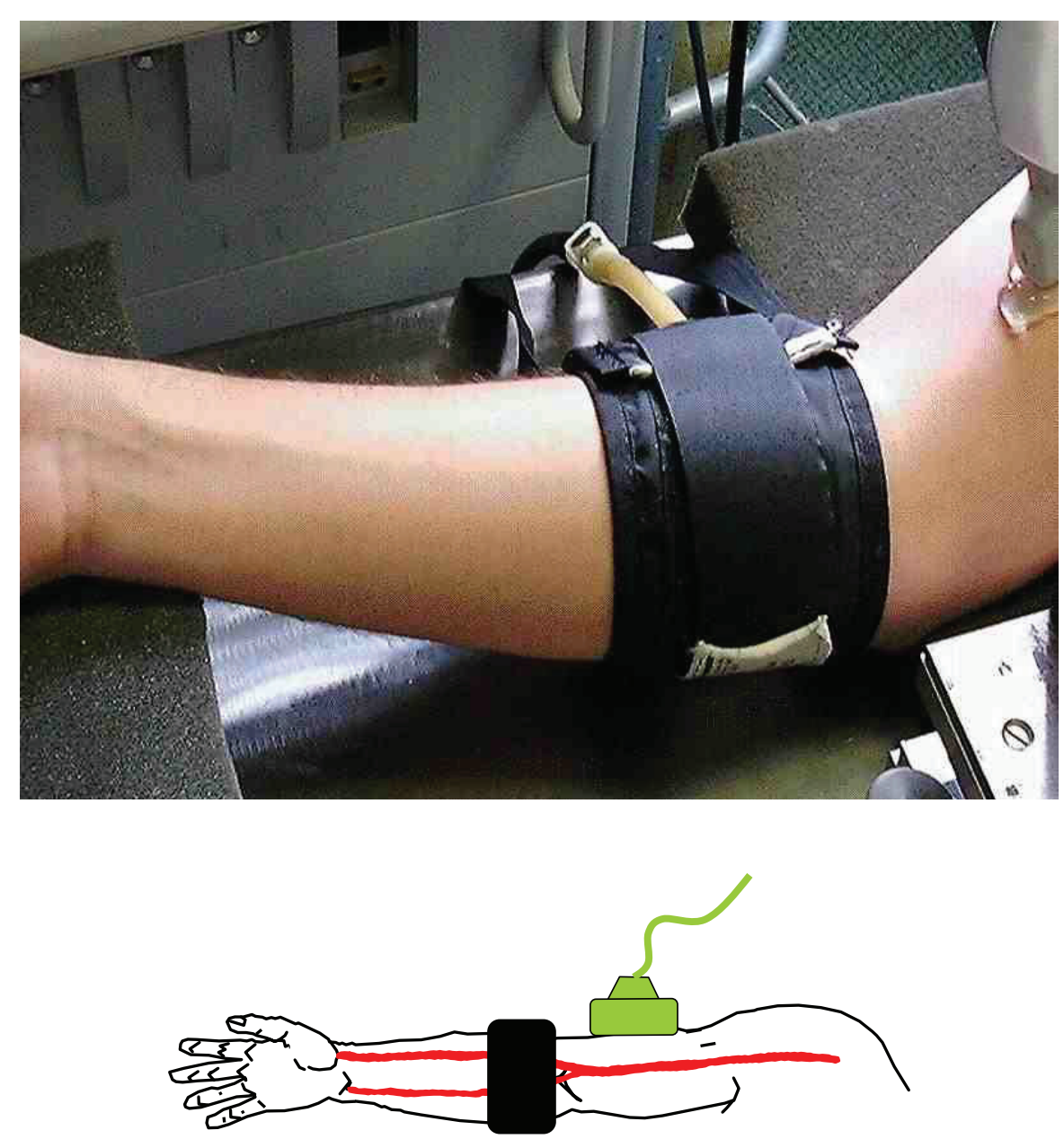

Figure I Probe position in relation to cuff. 
(Bots et al 2005). At present the lack of a scientific consensus on a standard protocol for measuring FMD precludes the accurate comparison of data between centers.

Guidelines for assessment of endothelial function in the brachial artery were published by an international task force (Corretti et al 2002); however these failed to come to an agreement as to the fundamental position of the cuff in relation to the ultrasound probe. This is recognized as the major difference between sites and produces discrepancies in FMD measurements in similar populations. In the interim, the working group on Endothelin and Endothelial Factors of the European Society of Hypertension (Deanfield et al 2005) have written a comprehensive update on endothelial function techniques. In addition, Donald and colleagues (2006) provide an explicit methodology for ultrasound FMD.

\section{Standardization of FMD methodology}

There are four critical elements of FMD methodology that need to be standardized:

- Probe position in relation to cuff.

- Shear stimulus (cuff occlusion time).

- Image measurement (stereotaxis and automation).

- Control of environmental factors.

\section{Probe position in relation to cuff}

The original method for brachial FMD described the cuff distal to the probe (Anderson and Mark 1989; Celermajer et al 1992), the cuff was placed around the forearm, and the brachial artery was imaged above the ante-cubital fossa.

Later, a second method developed with the cuff placed proximal to the imaging site. If the cuff is placed proximal to the transducer, the dilatation measured is greater, and, when this is the case, it is not clear what aspect of endothelial function is being measured (Berry et al 2000; Agewall et al 2001; Peretz et al 2007; Guthikonda et al 2007). NOdependent FMD measurement becomes confounded by super-added ischemic (NO-independent) vasodilatation (Doshi et al 2001).

Therefore, if the aim of the study is to assess NO bioavailability, the cuff should be distal in position to the probe (see Figure 1).

\section{Shear stimulus (cuff occlusion time)}

Varying lengths of cuff occlusion time have been used, but there is a general consensus that 5 minutes is the optimum time to elicit a good reactive hyperemia response and consequent dilatation. Studies have shown that the dilatatory response after prolonged cuff occlusion times (eg, 15 minutes) is not a pure NO-mediated event (Mullen et al 2001).

\section{Image measurement (stereotaxis and automation)}

With the introduction of electronic measurement for diameter change, it is imperative to have high-quality static images, which can only be acquired using micrometer-adjustable stereotactic apparatus (see Figure 4A in Deanfield et al 2007). Each image should be measured at end diastole, and the maximal dilation should be recorded rather than the dilation at any set time post cuff release; it has been shown that taking a measurement at a set time of 60 seconds, for example, misses the peak dilatation in approximately $70 \%$ of subjects (Palinkas et al 2002). Using this type of equipment and protocol can minimize inter-observer variability to within a range that is feasible for clinical application (Herrington et al 2001).

\section{Control of environmental factors}

Control of environmental factors is important for the longitudinal consistency of FMD measurements. The pertinent factors which are known to affect FMD measurements include room temperature, time of day, ingestion of fatty foods or caffeine, concurrent inflammation or infection, and stage of menstrual cycle (Hashimoto et al 1995; Leeson et al 1997; Williams et al 1999; Hingorani et al 2000; Duffey et al 2001; Charakida et al 2005).

\section{Is there any clinical value in measuring FMD?}

When performed by experienced personnel in a standardised setting with a rigid protocol, brachial artery FMD is a useful measure of NO-dependent endothelial function. If applied rigorously, FMD may serve as a marker for cardiac risk factors exposure and their functional biological effects in healthy volunteers (Celermajer et al 1994). However, screening for risk factors exposure is complicated by the fact that there is a wide variation in atheroscelorotic burden for similar risk factor exposure, and a considerable lag time between exposure to a risk factor and the manifestation of cardiovascular disease.

As a marker for preclinical disease, it has been suggested that FMD may be more closely correlated with the non-Framingham (ie, low or medium) risk factors than 
Framingham (high-level) risk factors (Witte et al 2005), however, in higher risk populations FMD has been investigated as a risk assessment tool and in patients having surgery for PVD, it is an independent predictor of cardiovascular events (Gokce et al 2003). In a recent study, Matsushima and colleagues (2007) claim that brachial artery FMD can diagnose the presence of coronary stenosis in high-risk individuals with similar accuracy to treadmill testing. Also, in small scale clinical studies, FMD has been found to be an independent predictor for further cardiac events in patients post myocardial infarction (Anderson et al 1995; Karatzis et al 2006).

Despite this clinical data, the acute, dynamic nature of the endothelium and its response to environmental factors gives rise to confounding results for a single FMD examination. If the environmental factors which are known to affect acutely endothelial function are not accounted for during testing, it is possible to get a false positive result for endothelial dysfunction, ie, a low FMD which may only be a transient state and not representative of pathology. As yet there is no standard protocol for the clinical application of FMD, and because single measurements can be so misleading, any clinical application would need to confirm endothelial dysfunction with repeat studies.

Presently, FMD remains a valuable and important research tool for studying populations rather than individuals. The additive value of brachial ultrasound FMD over and above established traditional clinical tools as yet remains to be proven, and ongoing studies continue to address this issue.

\section{Conclusion}

Validation of FMD continues in controlled research contexts. It is rapid and cheap. To date its reproducibility (and accuracy) as a tool to help in the management of asymptomatic and symptomatic individuals is unproven. Brachial ultrasound FMD allows the early detection of patients who would not be eligible for medical therapy under current guidelines, but who are at increased risk for cardiovascular events. There is no doubt that a test which detects otherwise (silent) cardiovascular risk would allow early intervention by lifestyle change, or drug therapy, and primary prevention could start as early as childhood (Celermajer and Ayer 2006). This in the long-term could dramatically reduce the mushrooming burden of cardiovascular disease on the healthcare system. However as FMD is technically demanding, implementation into a clinical setting would require standardized protocols and funding for recognized training within an established training pathway such as echocardiography or vascular technology.

It remains to be seen if the theoretical potential of ultrasound FMD as a research tool can ever be matched by its application in the less forgiving clinical environment.

\section{References}

Agewall S, Doughty RN, Bagg W, et al. 2001. Comparison of ultrasound assessment of flow-mediated dilatation in the radial and brachial artery with upper and forearm cuff positions. Clin Physiol, 21:9-14.

Anderson EA and Mark AL. 1989. Flow-mediated and reflex changes in large peripheral artery tone in humans. Circulation, 79:93-100.

Anderson TJ. 2007. Prognostic significance of brachial flow-mediated vasodilation. Circulation, 115:2373-75.

Berry KL, Skyrme-Jones RA, Meredith IT. 2000. Occlusion cuff position is an important determinant of the time course and magnitude of human brachial artery flow-mediated dilation. Clin Sci (Lond), 99:261-7.

Bonetti PO, Pumper GM, Higano ST et al. 2004. Noninvasive identification of patients with early coronary atherosclerosis by assessment of digital reactive hyperemia. J Am Coll Cardiol, 44:2137-41.

Bots M, Westerink J, Rabelink TJ, et al. 2005. Assessment of flow-mediated vasodilatation (FMD) of the brachial artery: effects of technical aspects of the FMD measurement on the FMD response. Eur Heart J, 26:363-8.

Celermajer DS, Sorensen KE, Gooch VM, et al. 1992. Non-invasive detection of endothelial dysfunction in children and adults at risk of atherosclerosis. Lancet, 340:1111-15.

Celermajer DS, Sorensen KE, Bull C, et al. 1994. Endothelium-dependent dilation in the systemic arteries of asymptomatic subjects relates to coronary risk factors and their interaction. $\mathrm{J} \mathrm{Am} \mathrm{Coll} \mathrm{Cardiol,}$ 24:1468-74.

Celermajer DS, Ayer JG. 2006. Childhood risk factors for adult cardiovascular disease and primary prevention in childhood. Heart, 92:1701-6.

Chong WC, Collins P, Webb CM, et al. 2006. Comparison of flow characteristics and vascular reactivity of radial artery and long saphenous vein grafts. $J$ Cardiothorac Surg, 1:4.

Charakida M, Donald AE, Terese M, et al. 2005. Endothelial dysfunction in childhood infection. Circulation, 111:1660-5.

Clarkson P, Adams MR, Powe AJ et al. 1996. Oral L-arginine improves endothelium-dependent dilation in hypercholesterolemic young adults. J Clin Invest, 97:1989-94.

Cooke JP, Dzau VJ. 1997. Nitric oxide synthase: role in the genesis of vascular disease. Annu Rev Med, 48:489-509.

Corretti MC, Anderson TJ, Benjamin EJ, et al. 2002. Guidelines for the ultrasound assessment of endothelial-dependent flow-mediated vasodilation of the brachial artery: a report of the International Brachial Artery Reactivity Task Force. J Am Coll Cardiol, 39:257-65.

Corson MA, James NL, Latta SE, et al. 1996. Phosphorylation of endothelial nitric oxide synthase in response to fluid shear stress. Circ Res, 79:984-91.

Deanfield J, Donald A, Ferri C, et al. 2005. Endothelial function and dysfunction. Part 1: Methodological issues for assessment in the different vascular beds: A statement by the Working Group on Endothelin and Endothelial Factors of the European Society of Hypertension. J Hypertens, 23:7-17.

Deanfield JE, Halcox JP, Rabelink TJ. 2007. Endothelial function and dysfunction: testing and clinical relevance. Circulation, 115:1285-95.

Donald AE, Charakida M, Cole TJ, et al. 2006. Non-invasive assessment of endothelial function : which technique? J Am Coll Cardiol, 48:1846-50.

Doshi SN, Naka KK, Payne N, et al. 2001. Flow-mediated dilatation following wrist and upper arm occlusion in humans: the contribution of nitric oxide. Clin Sci (Lond), 101:629-35. 
Duffy SJ, Keaney JF, Holbrook M, et al. 2001. Short- and long-term black tea consumption reverses endothelial dysfunction in patients with coronary artery disease. Circulation, 104:151-6.

Ford I, Murray H, Packard CJ, et al. 2007. Long-term follow-up of the West of Scotland Coronary Prevention Study. $N$ Engl J Med, 357:1477-86.

Förstermann U, Münzel T. 2006. Endothelial nitric oxide synthase in vascular disease: from marvel to menace. Circulation, 113:1708-14.

Furchgott RF, Zawadzki JV. 1980. The obligatory role of endothelial cells in the relaxation of arterial smooth muscle by acetylcholine. Nature, 288:373-76.

Gokce N, Keaney JF, Hunter LM, et al. 2003. Predictive value of noninvasively determined endothelial dysfunction for long-term cardiovascular events in patients with peripheral vascular disease. $\mathrm{J} \mathrm{Am} \mathrm{Coll}$ Cardiol, 41:1769-75.

Guthikonda S, Sinkey CA, Haynes WG. 2007. What is the most appropriate methodology for detection of conduit artery endothelial dysfunction? Arterioscler Thromb Vasc Biol, 27:1172-6.

Halcox JP, Schenke WH, Zalos G, et al. 2002. Prognostic value of coronary vascular endothelial dysfunction. Circulation, 106:653-8.

Hansell J, Henareh L, Agewall S, et al. 2004. Non-invasive assessment of endothelial function - relation between vasodilatory responses in skin microcirculation and brachial artery. Clin Physiol Funct Imaging, 24:317-22.

Hashimoto M, Akishita M, Eto M, et al. 1995. Modulation of endotheliumdependent flow-mediated dilatation of the brachial artery by sex and menstrual cycle. Circulation, 92:3431-5.

Herrington DM, Fan L, Drum M, et al. 2001. Brachial flow-mediated vasodilator responses in population-based research: methods, reproducibility and effects of age, gender and baseline diameter. $J$ Cardiovasc Risk, 8:319-28.

Hingorani AD, Cross J, Kharbanda RK, et al. 2000. Acute systemic inflammation impairs endothelium-dependent dilatation in humans. Circulation, 102:994-9.

Juonala M, Viikari JSA, Laitinen T, et al. 2004. Interrelations between brachial endothelial function and carotid intima-media thickness in young adults. The cardiovascular risk in young Finns study. Circulation, 110:2918-23.

Kharbanda RK, Deanfield JE. 2001. Functions of the healthy endothelium. Coron Artery Dis, 12:485-91.

Karatzis EN, Ikonomidis I, Vamvakou GD, et al. 2006. Long-term prognostic role of flow-mediated dilatation of the brachial artery after acute coronary syndromes without ST elevation. Am J Card, 98:1424-8.

Leeson CPM, Whincup PH, Cook DG, et al. 1997. Flow-mediated dilation in 9- to 11-year-old children: the influence of intrauterine and childhood factors. Circulation, 96:2233-8.

Leeson CP, Robinson M, Francis JM, et al. 2006. Cardiovascular magnetic resonance imaging for non-invasive assessment of vascular function: validation against ultrasound. J Cardiovasc Magn Reson, 8:381-7.
Matsushima Y, Takase B, Uehata A, et al. 2007. Comparative predictive and diagnostic value of flow-mediated vasodilation in the brachial artery and intima media thickness of the carotid artery for assessment of coronary artery disease severity. Int J Cardiol, 117:165-72.

Mullen MJ, Kharbanda RK, Cross J, et al. 2001. Heterogeneous nature of flow-mediated dilatation in human conduit arteries in vivo: relevance to endothelial dysfunction in hypercholesterolaemia. Circ Res, 88:145-51.

Nicholls SJ, Sipahi I, Schoenhagen P, et al. 2006. Intravascular ultrasound assessment of novel antiatherosclerotic therapies: rationale and design of the Acyl-CoA: Cholesterol Acyltransferase Intravascular Atherosclerosis Treatment Evaluation (ACTIVATE) Study. Am Heart J, 152:67-74.

Palinkas A, Toth E, Venneri L, et al. 2002. Temporal heterogeneity of endothelium-dependent and -independent dilatation of brachial artery in patients with coronary artery disease. Int J Cardiovasc Imaging, 18:337-42.

Peretz A, Leotta DF, Sullivan JH, et al. 2007. Flow mediated dilation of the brachial artery: an investigation of methods requiring further standardisation. BMC Cardiovasc Disord, 7:11.

Pohl U, Holtz J, Busse R, et al. 1986. Crucial role of endothelium in the vasodilator response to increased flow in vivo. Hypertension, 8:37-44.

Reilly JJ, Armstrong J, Dorosty AR, et al. 2005. Early life risk factors for obesity in childhood: cohort study. BMJ, 330:1357.

Rubanyi GM, Romero JC, Vanhoutte PM. 1986. Flow-induced release of endothelium-derived relaxing factor. Am J Physiol, 250(6 Pt 2):H1145-9.

Sankatsing RR, de Groot E, Jukema JW, et al. 2005. Surrogate markers for atherosclerotic disease. Curr Opin Lipidol, 16:434-41.

Schechter AN, Gladwin MT. 2003. Haemoglobin and the paracrine and endocrine functions of nitric oxide. $N$ Engl J Med, 384:1483-5.

Shimbo D, Grahame-Clarke C, Miyake Y, et al. 2007. The association between endothelial dysfunction and cardiovascular outcomes in a population-based multi-ethnic cohort. Atherosclerosis, 192:197-203

Smiesko V, Kozik J, Dolezel S. 1985. Role of endothelium in the control of arterial diameter by blood flow. Blood Vessels, 22:247-51.

Strain WD, Chaturvedi N, Leggetter S, et al. 2005. Ethnic differences in skin microvascular function and their relation to cardiac target-organ damage. J Hypertens, 23:133-40.

Suessenbacher A, Frick M, Alber HF, et al. 2006. Association of improvement of brachial artery flow-mediated vasodilation with cardiovascular events. Vasc Med, 11:239-44.

Williams MJ, Sutherland WH, McCormick MP, et al. 1999. Impaired endothelial function following a meal rich in used cooking fat. $J \mathrm{Am}$ Coll Cardiol, 33:1050-5.

Witte DR, Westerlink J, de Koning EJ, et al. 2005. Is the association between flow-mediated dilation and cardiovascular risk limited to low-risk populations? J Am Coll Cardiol, 45:1987-93.

Yeboah J, Crousse JR, Hsu F, et al. 2007. Brachial flow-mediated dilation predicts incident cardiovascular events in older adults: The Cardiovascular Health Study. Circulation, 115:2390-97. 